\title{
THE LATENT ROOTS OF A MATRIX OF SPECIAL TYPE
}

\section{BY JOHN WILLIAMSON}

1. Introduction. In this paper the latent roots of a matrix, each of whose elements are suitably restricted rational functions of a square matrix $A$, are discussed. In particular a theorem is proved about the latent roots of such a matrix, which reduces in the simplest case to the familiar theorem on the value of the latent roots of a rational function of a matrix $A$. The third section illustrates the fact that the values of circulant determinants, and of determinants composed of blocks which are themselves circulants, can be easily deduced from their matrix properties.

2. Latent Roots. Let $A$ be a square matrix of $n$ rows and $n$ columns and let $\lambda_{1}, \lambda_{2}, \cdots, \lambda_{n}$ be the latent roots of $A$, where the $\lambda_{i}$ need not be distinct. Then by a well known theorem on matrices* there exists a non-singular matrix $X$, which transforms $A$ into a matrix whose elements in the leading diagonal are the latent roots of $A$, while all the elements to the left of the leading diagonal are zero. This may be expressed by the matrix equation

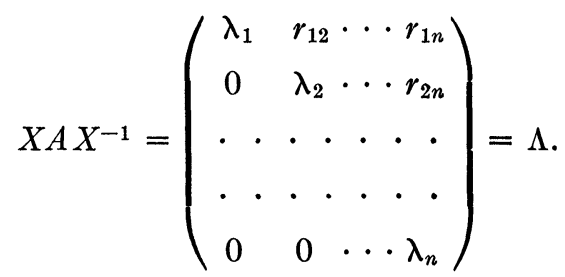

It follows from (1) that, if $f(A)$ is a rational function of the matrix $A$, of which the denominator is non-singular, the matrix $X$ transforms the matrix $f(A)$ into a matrix of the same type as $\Lambda$, where the elements in the leading diagonal are $f\left(\lambda_{1}\right)$, $f\left(\lambda_{2}\right), \cdots, f\left(\lambda_{n}\right)$. Or

$$
X f(A) X^{-1}=f(\Lambda)
$$

\footnotetext{
* A. Wintner, Spektraltheorie der unendlichen Matrizen, pp. 20-22.
} 
where the elements to the left of the leading diagonal in $f(\Lambda)$ are all zero. If now

$$
B=\left(A_{i j}\right), \quad(i, j=1,2, \cdots, m),
$$

denote an $m$-rowed square matrix all of whose elements are $n$-rowed square matrices $A_{i j}, B$ is a square matrix of order $m n$ in the elements of the matrices $A_{i j}$. If

$$
Y=\left(X_{i j}\right), \quad(i, j=1,2, \cdots, m),
$$

is a matrix of the same type as $B$, where $X_{i j}=0$, if $i \neq j$, and $X_{i i}=X$, then by simple multiplication

$$
Y B Y^{-1}=\left(X A_{i j} X^{-1}\right) .
$$

In particular, if $A_{i j}=f_{i j}(A)$ be rational functions, whose denominators are non-singular, of the original matrix $A$, then

$$
C=\left(f_{i j}(A)\right)=M[A]
$$

is an $n m$-rowed square matrix of the same type as $B$. It follows from equations (2), (4), and (5) that

$$
Y C Y^{-1}=\left(X f_{i j}(A) X^{-1}\right)=\left(f_{i j}(\Lambda)\right)=D .
$$

Accordingly the latent roots of $C$ are the same as the latent roots of $D$. But the latent roots of $D$ are the roots of the equation $|D-\lambda E|=0$, obtained by equating the determinant of the matrix $D-\lambda E$ to zero. If $\Delta$ denote the determinant of $D-\lambda E$ and if, for brevity, the element in the $i$ th row and the $j$ th column of $\Delta$ is indicated by $(i, j)$, it follows from (2) and (6) that (7) $\begin{aligned} & (s n+a, r n+i)=0, \text { if } a>i, \quad(r, s=0,1, \cdots, m-1), \\ & (s n+i, r n+i)=f_{s+1, r+1}\left(\lambda_{i}\right)-\delta_{s}{ }^{r} \lambda, \quad(i=1,2, \cdots, n) .\end{aligned}$ From $\Delta$ a new determinant $\Delta^{\prime}$ is formed by taking for the rows and columns of $\Delta^{\prime}$ in succession the

$$
\begin{aligned}
& \text { 1st, }(n+1) \text { th, }(2 n+1) \text { th, } \cdots,(n(m-1)+1) \text { th, } \\
& 2 \mathrm{~d}, \quad(n+2) \text { th, }(2 n+2) \text { th, } \cdots,(n(m-1)+2) \text { th, } \\
& n \text { th, } \quad 2 n \text { th, } \quad 3 n \text { th, } \cdots, \quad m n \text { th, }
\end{aligned}
$$

rows and columns of $\Delta$. Thus, by (7), $\Delta^{\prime}$ is a determinant in which all the elements in the first $m$ columns vanish except 
those in the first $m$ rows; all the elements in the second $m$ columns vanish except those in the first $2 m$ rows, and in general all the elements in the $i$ th set of $m$ columns vanish except those in the first im rows. Therefore $\Delta^{\prime}$ is the product of the $n$ determinants formed from the first $m$ rows and the first $m$ columns, from the second $m$ rows and the second $m$ columns, etc. But by (7) the determinant formed from the $k$ th set of $m$ columns, and the $k$ th set of $m$ rows of $\Delta^{\prime}$ is

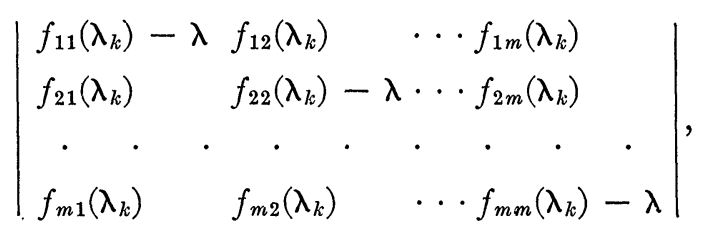

or more shortly $\left|f_{r s}\left(\lambda_{k}\right)-\delta_{r}{ }^{8} \lambda\right|,(r, s=1,2, \cdots, m)$. Hence with the notation of (5)

$$
\Delta^{\prime}=\prod_{k=1}^{n}\left|M\left[\lambda_{k}\right]-\lambda E\right|
$$

But, as $\Delta$ differs from $\Delta^{\prime}$ by at most a sign, $\Delta^{\prime}=0$ is equivalent to $\Delta=0$ and accordingly the latent roots of $D$ and therefore the latent roots of $C$ are the $n m$ roots of the $n$ equations obtained by equating the $n$ factors $\left|M\left[\lambda_{k}\right]-\lambda E\right|$ of (8) in turn to zero. Since the roots of the equation $\left|M\left[\lambda_{k}\right]-\lambda E\right|=0$ are the latent roots of the matrix $M\left[\lambda_{k}\right]$, the following theorem is true.

THEOREM 1. If $A$ be an n-rowed square matrix, whose latent roots are $\lambda_{1}, \lambda_{2}, \cdots, \lambda_{n}$ and $C=\left(f_{r s}(A)\right)$ be a square matrix whose $m^{2}$ elements are themselves $n$-rowed square matrices, which are rational functions, with non-singular denominators, of the original matrix $A$, then the latent roots of the matrix $C$ are the $n m$ latent roots of the $n$ m-rowed square matrices $\left(f_{r s}\left(\lambda_{k}\right)\right),(k=1,2, \cdots, n)$.

COROLlaRY 1. If $m=1$, this theorem reduces to the well known theorem that the latent roots of $f_{11}(A)$ are $f_{11}\left(\lambda_{k}\right)$, for the latent root of the one-rowed square matrix $f_{11}\left(\lambda_{k}\right)$ is obviously $f_{11}\left(\lambda_{k}\right)$.

Corollary 2. If $n=1$, the square matrix $A$ may be taken to be the number 1 and the latent root of 1, considered as a matrix, as 1 itself, so that in this case the theorem simply reduces to the definition of the latent roots of a matrix. 
If $F(M[\lambda])=N[\lambda]=\left(g_{r s}(\lambda)\right)$ is a matrix polynomial in the matrix $M[\lambda]$, it is easily shown that $F(C)=N[A]=\left(g_{r s}(A)\right)$. Hence by Theorem 1 the latent roots of $F(C)$ are the $n m$ latent roots of the $n m$-rowed matrices $F\left(M\left[\lambda_{k}\right]\right),(k=1,2, \cdots, n)$. In fact if $F(C)$ is a rational function of $C$ of which the denominator $H(C)$ is non-singular, the latent roots of $F(C)$ are the $n m$ latent roots of the $n$ matrices $F\left(M\left[\lambda_{k}\right]\right)$. But, the determinant of $H(C)$ is the product of the latent roots of $H(C)$ and therefore is equal to the product of the determinants of the $n$ matrices $H\left(M\left[\lambda_{k}\right]\right)$. Hence $H(C)$ is non-singular if the $n$ matrices $H\left(M\left[\lambda_{k}\right]\right)$ are nonsingular. Accordingly this proves the following theorem.

Theorem 2. If $A$ and $C$ are the matrices of Theorem 1 and if $F(C)$ is a rational function of $C$ with a non-singular denominator, the latent roots of $F(C)$ are the $n m$ latent roots of the $n$ matrices $F\left(M\left[\lambda_{k}\right]\right),(k=1,2, \cdots, n)$. Moreover, the denominator of $F(C)$ is non-singular if and only if the denominators of all the matrices $F\left(M\left[\lambda_{k}\right]\right)$ are non-singular.

Theorem 1, though perhaps presented in its most interesting form, is a particular case of a more general theorem. For the proof of Theorem 1 it is not essential that the functions $f_{i j}(A)$ be functions of the same matrix $A$. In fact for $f_{i j}(A)$ in Theorem 1 we may write $f_{i j}\left(A_{i j}\right)$ without essentially altering the proof, provided that the same matrix $X$ transforms all the matrices $A_{i j}$ into matrices of type $\Lambda$. Thus we have the following theorem.

TheOREM 3. If $A_{i j}$ is an n-rowed square matrix, whose latent roots are $\lambda_{i j}^{(1)}, \lambda_{i j}^{(2)}, \cdots, \quad \lambda_{i j}^{(n)}$, and if for all values of $i, j=1$, $2, \cdots, m$ the same non-singular matrix $X$ transforms the matrix $A_{i j}$ into a matrix of type $\Lambda$ with $\lambda_{i j}^{(1)}, \lambda_{i j}^{(2)}, \cdots, \lambda_{i j}^{(n)}$ as its leading diagonal, then the latent roots of the matrix

$$
D=\left(f_{i j}\left(A_{i j}\right)\right), \quad(i, j=1,2, \cdots, m),
$$

are the $n m$ latent roots of the $n m$-rowed square matrices

$$
\left(f_{i j}\left(\lambda_{i j}^{(k)}\right)\right), \quad(k=1,2, \cdots, n) .
$$

3. Applications. Let $e_{i j},(i, j=1,2, \cdots, n)$ denote the matrix whose element in the $i$ th row and the $j$ th column is 1 , while all other elements are zero. Further let $e_{k s}$ denote $e_{i j}$ if $k>n, s>n$, but $k \equiv i$ and $s \equiv j \bmod n$. Thus the matrices $E_{r}$ defined by 


$$
E_{r}=\sum_{i=1}^{n} e_{i, i+r}=e_{\alpha, \alpha+r}
$$

where the $\alpha$ 's are summation suffixes, are non-singular matrices. Since the matrices $e_{i j}$ obey the rule of multiplication, we have

$$
\begin{aligned}
e_{i j} e_{k m} & =\delta_{j}^{k} e_{i m}, \\
E_{r} E_{s} & =e_{\alpha, \alpha+r} e_{\beta, \beta+s}=e_{\alpha, \alpha+r+s}=E_{r+s} .
\end{aligned}
$$

Hence by repeated applications of (11) with $s=1$ and $r=1$, $2, \cdots, r-1$,

$$
E_{1}^{r}=E_{r}, E_{1}^{n}=E_{0}=E
$$

the unit matrix.

If now

$$
f(x)=a_{0}+a_{1} x+a_{2} x^{2}+\cdots+a_{n-1} x^{n-1},
$$

by (12)

$$
f\left(E_{1}\right)=a_{0} E_{0}+a_{1} E_{1}+a_{2} E_{2}+\cdots+a_{n-1} E_{n-1} .
$$

But by (9)

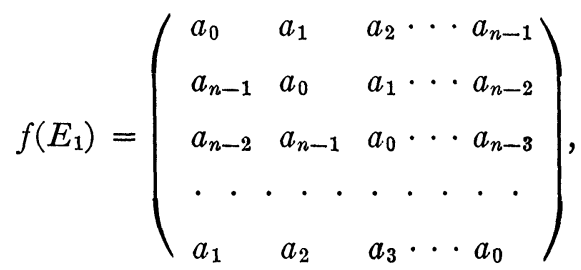

that is, $f\left(E_{1}\right)$ is a matrix whose determinant is a circulant and may be for brevity called a circulant matrix. If $f_{i j}(x)=\sum_{k=0}^{n-1} a_{k}^{i j} x^{k}$ and $A=E_{1}$, the matrix $C$ defined by (5) is a matrix whose component matrices $f_{i j}\left(E_{1}\right)$ are all circulant matrices. By Theorem 1 the latent roots of $C$ are the $n m$ latent roots of the matrices $M\left[\lambda_{k}\right]$, where the $\lambda_{k}$ are the latent roots of $E_{1}$. But the latent roots of $E_{1}$ are the $n$th roots of unity $\omega_{1}, \omega_{2}, \cdots, \omega_{n}$. Hence the latent roots of $C$ are the $n m$ latent roots of the $n$ matrices $M\left[\omega_{k}\right], k=1,2, \cdots, n$. In particular the determinant of $C$ is the product of the latent roots of $C$ so that

$$
|C|=\prod_{k=1}^{n}\left|M\left[\omega_{k}\right]\right|=\prod_{k=1}^{n}\left|f_{i j}\left(\omega_{k}\right)\right|
$$


If $m=1$ in (13), this result reduces to the well known theorem on the value of a circulant, ${ }^{*}$ while if $m>1$ formula (13) is the statement of a result proved by Zariski on the value of a determinant composed of circulants. $\dagger$

If, with the previous interpretation of the notation $e_{i j}$, we define

then

$$
E_{1}=\sum_{i=1}^{n} x_{i} e_{i, i+1}
$$

$$
E_{1}^{r}=\sum_{i=1}^{n} x_{i} x_{i+1} \cdots x_{i+r-1} e_{i, i+r},
$$

where $x_{i}=x_{j}$, if $i \equiv j \bmod n$, and Theorem 1 gives the value of another type of determinant, which, for $n=4$, is the determinant of the matrix

(14) $f\left(E_{1}\right)=\left(\begin{array}{llll}a_{0} x_{1} x_{2} x_{3} x_{4} & a_{1} x_{1} & a_{2} x_{1} x_{2} & a_{3} x_{1} x_{2} x_{3} \\ a_{3} x_{2} x_{3} x_{4} & a_{0} x_{2} x_{3} x_{4} x_{1} & a_{1} x_{2} & a_{2} x_{2} x_{3} \\ a_{2} x_{3} x_{4} & a_{3} x_{3} x_{4} x_{1} & a_{0} x_{3} x_{4} x_{1} x_{2} & a_{1} x_{3} \\ a_{1} x_{4} & a_{2} x_{4} x_{1} & a_{3} x_{4} x_{1} x_{2} & a_{0} x_{4} x_{1} x_{2} x_{3}\end{array}\right)$.

Since the latent roots of $E_{1}$ are now $\rho_{k}$, where the $\rho_{k}$ are the $n$th roots of the product $x_{1} x_{2}, \cdots, x_{n}$, the value of the determinant of such a matrix is $\prod_{k=1}^{n} f^{\prime}\left(\rho_{k}\right)$, where $f^{\prime}(x)$ is the polynomial $f(x)$ with its constant term replaced by $a_{0} x^{n}$. If $E_{1}=A$, but $m>1$, Theorem 1 gives the value of a determinant composed of $m^{2}$ blocks of the type (14), where the $a_{i}$ may vary from block to block, but the $x_{i}$ must remain the same.

The Johns Hopkins University

* Thomas Muir, Theory of Determinants, vols. 1, 2, 3. Under the heading of circulants many determinants which are special cases of Zariski's are discussed.

$\dagger \mathrm{O}$. Zariski, On the linear connection index of the algebraic surfaces $z^{n}=f(x, y)$, Proceedings of the National Academy of Sciences, vol. 15 (1929), No. 6, p. 497. 